Z. klin. Chem. u. klin. Biochem.

7. Jg., S. 590-591, November 1969

\title{
Messung der Glucoseoxydation an der isoliert perfundierten Rattenleber mit Glucose- $\left[6{ }^{3} \mathrm{H}\right]$
}

\author{
Von P. Glogner und H. J. SChUrek \\ Auss der Mediqinischen Universitätsklinik Tü̈bingen (Direktor: Prof. Dr. Dr. b. c. H. E. Bock) und der Medizinischen \\ Universitäts-Poliklinik Marburg/Labn (Komm. Direktor: Priv.-Doz. Dr. D. Voss)
}

(Eingegangen am 27. Juni 1969)

Es wird eine Methode zur Messung der Glucose-Oxydationsrate an isoliert perfundierten Rattenlebern bescbrieben: Glucose-[6- $\left.{ }^{3} \mathrm{H}\right]$ wird durch den Leberstoffwechsel zu HTO oxydiert. HTO wird durch Gefrierdestillation isoliert und die Tritiumaktivität im Flüssigkeitsszintillationszähler gemessen. Die Ergebnisse zeigen, daß Glucose von der Leber nur zu einem sehr geringen Umfang oxydiert wird.

\section{The measurement of glucose oxidation in isolated, perfused rat liver, with the aid of $\left[6-{ }^{3} \mathrm{H}\right]$ glucose}

A method is described for the measurement of the rate of oxidation of glucose in isolated, perfused rat liver. The oxidation of [6- $\left.{ }^{3} \mathrm{H}\right]-$ glucose by the liver produces HTO, which is isolated by freeze-destillation, prior to the determination of tritium activity in a liquid scintillation counter. The results show that glucose is oxidised by the liver only to a very small extent.

Bei der Nessung der Glucoseoxydation an isoliert perfundierterı Rattenlebern treten Schwierigkeiten auf: Setzt man Glucose-[6-14 $\mathrm{C}]$ als Substrat ein und bestimmt die Ausbeute an ${ }^{14} \mathrm{CO}_{2}$, werden zu niedrige Oxydationsraten gemessen. Ursache dafür ist wahrscheinlich ein Entweichen erheblicher $\mathrm{CO}_{2}$-Mengen durch Siliconschläuche und die Leberkapsel (1). Dieser Nachteil macht ein absolut gasdichtes System erforderlich. Demgegenüber bleibt $\mathrm{H}_{2} \mathrm{O}$ im Perfusionsmedium (2). Es lag somit nahe, Glucose-[6- $\left.{ }^{3} \mathrm{H}\right]$ als Substrat zu verwenden und die Menge gebildeten Tritium-Wassers (HTO) zu messen. Beim Einsatz von Glucose- $\left[6-{ }^{3} \mathrm{H}\right]$ bleibt das Isotop über die Glykolyse bis zur Stufe der Citronensäure gebunden und wird dann auf Grund theoretischer Erwägungen (3) beim Übergang zu Isocitronensäure mit zellulärem Wasser teilweise ausgetauscht. Die übrigen Dehydrierungsreaktionen des Tricarbonsäurezyklus in Verbindung mit der Atmung liefern HTO.

$\mathrm{Da}$ schließlich jedes Glucosemolekül, welches über den Tricarbonsäure-Zyklus oxydativ umgesetzt wird, Wasser bildet, lautet die Summenformel:

$$
\text { Glucose- }[6-3 \mathrm{H}]+6 \mathrm{O}_{2}=6 \mathrm{CO}_{2}+\mathrm{HTO}+5 \mathrm{H}_{2} \mathrm{O}
$$

$\mathrm{Da}$ im Perfusionssystem eine Verdünnung der Glucose[6- $\left.{ }^{3} \mathrm{H}\right]$-Aktivität durch das Leberglykogen und die Gluconeogenese eintritt, muß die spezifische Aktivität der Glucose im Medium ermittelt werden.

\section{Material und Methoden}

Operationstechnik und Präparation der Rattenlebern siehe (2). Das Perfusionsmedium aus standardisierter Salzlösung mit Rindererythrocyten wurde von SCHIMASSEK (4) angegeben:

D-Glucose- $\left[6-{ }^{3} \mathrm{H}\right]$ wurde vom Radiochemical Centre Amersham/ England über die Fa. Buchler, Braunschweig, bezogen und jeweils in einer Menge von $140-240 \mu \mathrm{C}$ dem Medium als wäßr. Lösung zugesetzt.

Die enzymatische Bestimmung von Glucose mit Hexokinase (EC 2.7.1.1)/Glucose-6-phosphatdehydrogenase (EC 1.1.1.49) wurde mit Testpackungen der Fa. Boehringer, Mannheim, durchgeführt (2).
Isolierung der Glucose aus Perchlorsäureextrakten (0,3M Endkonzentration): Absteigende Papierchromatographie in Butanol/ Eisessig/Wasser (4:1:5 v/v), $R_{\mathrm{F}}$-Wert für Glucose $=0,18$. Anfärbung der Glucosebande mit Anilinphosphat. Markierung im UVLicht, Ausschneiden der Flecken, Elution in Szintillationsflüssigkeit (s. u.) und direkte Messung im Flüssigkeitsszintillationszähler der $\mathrm{Fa}$. Packard gegen inneren Standard.

HTO-Isolierung: Schnelles Einfrieren von $0,3 \mathrm{ml}$ perchlorsaurem Extrakt durch Aceton/Kohlensäure-Eis im Rundkolben, Evakuierung auf 3-4 Torr, Wechsel der Kältemischung auf ein parallel geschaltetes Spitzkölbchen und vorsichtiges Auftauen im Rundkolben. Flüchtige Bestandteile wie $\mathrm{H}_{2} \mathrm{O}$ und $\mathrm{HTO}$ treten über, desgleichen Perchlorsäure, die den $\mathrm{pH}$-Wert auf etwa 1 senkt. Lactat, Pyruvat usw. sind unter diesen Bedingungen nicht flüchtig. Durch Verwendung eines inneren Standards ist der pH-Effekt zu vernachlässigen.

Messung der Destillataktivität: $0,1 \mathrm{~m} /$ werden in $15 \mathrm{~m} /$ Szintillator aufgenommen $\left(4 \mathrm{~g} / / \mathrm{PPO}^{1}\right)$ und $\left.100 \mathrm{mg} / / \mathrm{POPOP}^{1}\right)$, Packard in Toluol/Äthanol, $2+1(v+v))$ und direkt gemessen. Nennenswerte Aktivitäten in anderen Substanzflecken außer Glucose waren beim Durchmessen der Chromatogramme nicht nachzuweisen.

\section{Ergebnisse}

Während der Perfusion steigt der Glucosespiegel im Perfusatinitial stark an. Nach 15 Min. wird der weitere Anstieg linear (Abb. 1).

Im Mittel werden in 2 Stdn. bei einem Lebergewicht von $8 \mathrm{~g}$ etwa $120 \mathrm{mg}$, d. h. $0,7 \mathrm{mMol}$ Glucose mehr ausgeschüttet als verwertet $(9 \mathrm{mMol} / \mathrm{l}$ bei einem Perfusionsvolumen von $130 \mathrm{ml}$ ).

Dementsprechend sinkt durch Verdünnung die mittlere spezifische Aktivität ab (Abb. 2, oben).

Die Aktivitäten von HTO im Destillat steigen kontinuierlich an (Abb. 2, unten), wobei sich größere individuelle Schwankungen ergeben.

Die absolute Bildung von HTO errechnet sich zu

$$
\frac{\Delta \text { HTO }}{\text { mittl. spezif. Akt./Zeit }}
$$

1) Abkürzungen: $\mathrm{PPO}=2,5$ Diphenyloxazol, $\mathrm{POPOP}=1,4$-bis2-(4-Methyl-5-phenyloxazolyl)-benzol. 


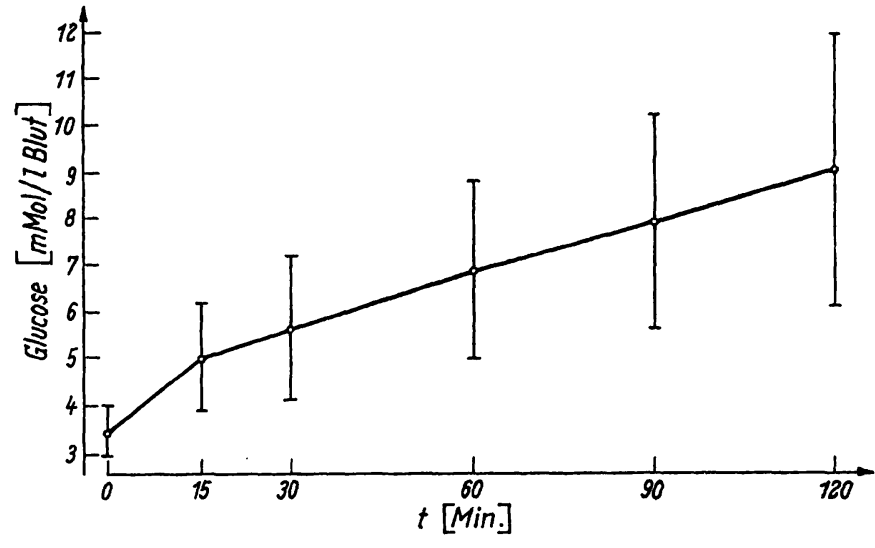

Abb. 1

Glucosekonzentration im Perfusionsmedium. Mittelwerte mit Standardabweichung, $n=8$. Endvolumen des Perfusats im Mittel $130 \mathrm{ml}$

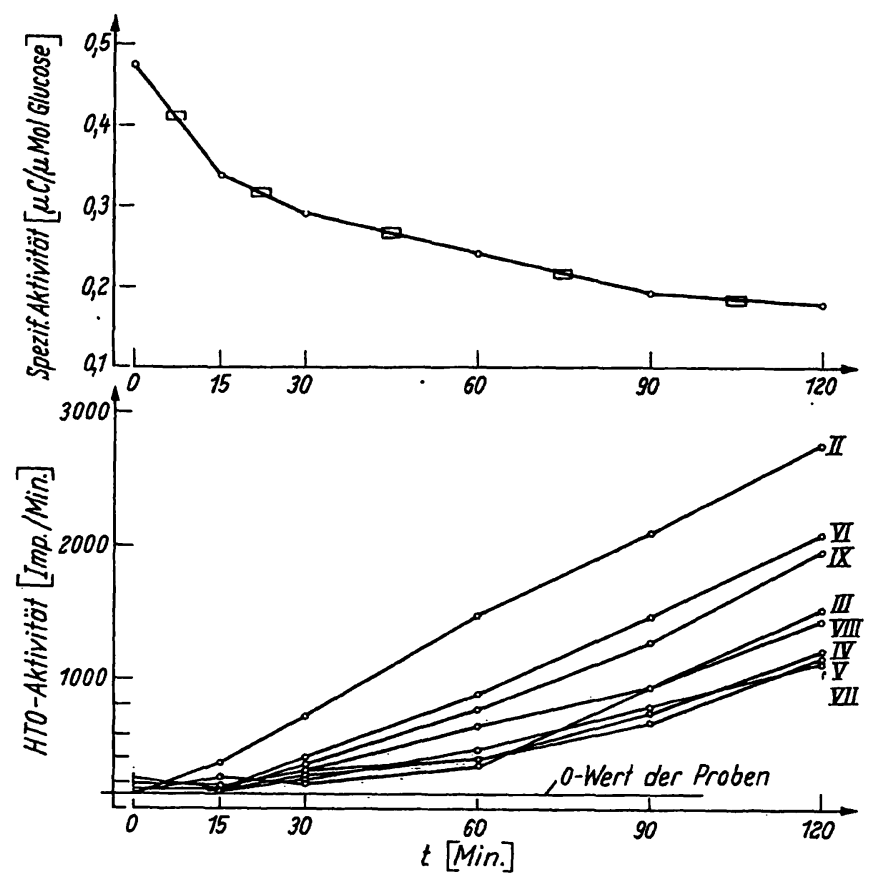

Abb. 2

Verhalten der spezifischen Glucoseaktivitäten im Medium (oben, Aktivität pro $0,1 \mathrm{ml}$ Destillat $=0,195 \mathrm{ml}$ Blut (unten), Werte aus 8 Einzelperfusionen

Abbildung 3 zeigt die absoluten Aktivitäten im Mittel wie die summierten Zuwachsraten an HTO in den einzelnen Versuchen.

Bei 2 Versuchen kam es zu einem sehr starken Anstieg. Hier lagen auch stark überhöhte Glụcosespiegel im Perfusionsmedium vor. Demnach besteht wahrscheinlich ein Zusammenhang zwischen Glucosespiegel und Oxydationsrate.

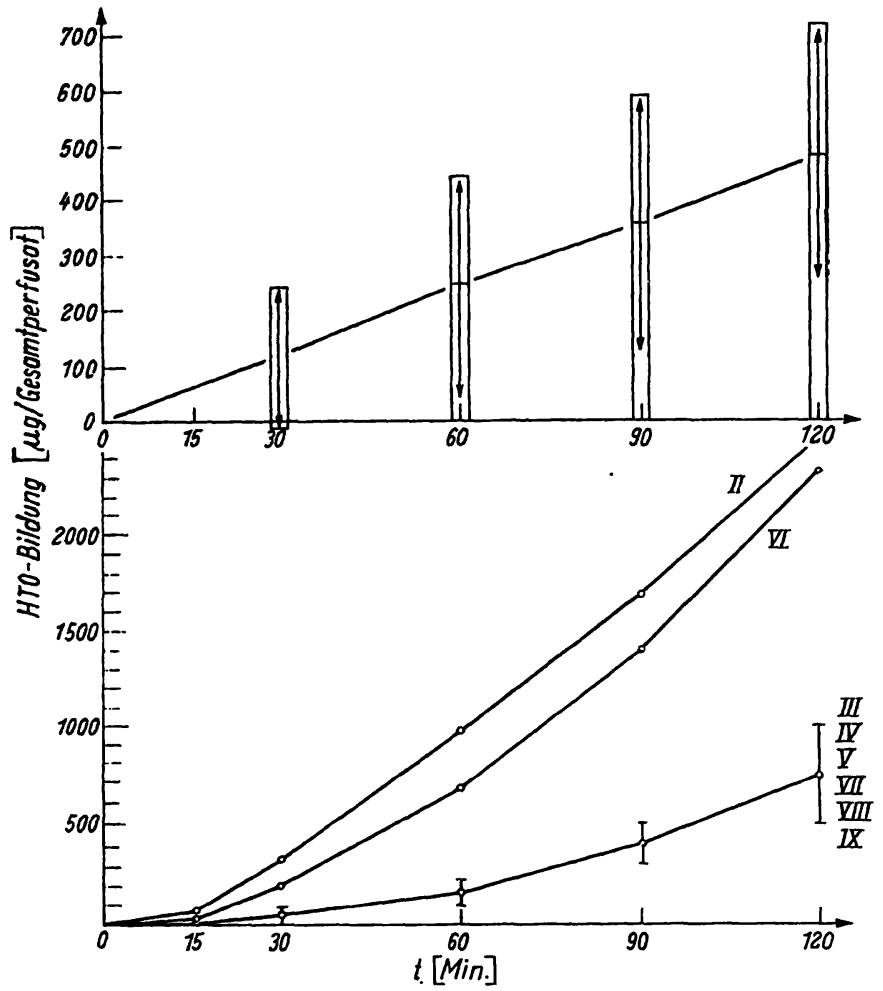

Abb. 3

Absolutwerte von HTO im Gesamtperfusat $(=160 \mathrm{ml}) . \mathrm{n}=8$, Mittelwerte mit Standardabweichung. Unten: Summierte HTO-Zuwachsraten aus 6 Einzelperfusionen sowie HTO-Zuwachsraten zweier davon abweichender Einzelperfusionen

Tab. 1

Anteil der Glucoseoxydation am $\mathrm{O}_{2}$-Verbrauch der Leber. Mittelwerte aus 6 Versuchen. Untere Gruppe aus Abbildung 3 unten

\begin{tabular}{ccc}
\hline $\begin{array}{c}\text { Zeitraum } \\
\text { (Minuten) }\end{array}$ & $+\mu$ Mol HTO/g & $\begin{array}{c}\text { \% Glucoseoxydation vom } \\
\text { Gesamt-O }\end{array}$ \\
\hline $30-60$ & 0,032 & 1,5 \\
$60-90$ & 0,056 & 2,5 \\
$90-120$ & 0,089 & 4,0 \\
\hline
\end{tabular}

Der Sauerstoffverbrauch der Rattenleber beträgt, unter gleichen Bedingungen gemessen (4), $2,2 \mu \mathrm{Mol} \mathrm{O}_{2} / \mathrm{g}$ Leberfrischgewicht/Min. Setzt man die HTO-Bildung aus Glucose in Beziehung zu diesem als konstant ermitteltem $\mathrm{O}_{2}$-Verbrauch $\left(2,2 \mu \mathrm{Mol} / \mathrm{g} / \mathrm{Min} . \mathrm{O}_{2}=100 \%\right)$ so ergibt sich (Tab. 1), daß die Leber nur in sehr geringem Maße Glucose verbrennen kann.

Wie bereits von Schrmassex (5) gezeigt, dürfte die Glucose als Substrat für die Energiegewinnung der Leber kaum ins Gewicht fallen.

Dex Deutschen Forschungsgemeinschaft sei an dieser Stelle für die Unterstützung der Arbeit gedankt.

\section{Literatur}

1. Miller, L. L., C. B. Bly, M. L. Wabon und W. F. Bale, J. exper. Med. 94, 431 (1951). - 2. GLOGNER, P., H. NIETh, H. WAGNER und K. GLOEDE, Klin. Wschr. 44, 1040 (1966). - 3. BrodA, E., Radioaktive Isotope in der Biochemie. F. Deuticke Verlag, Wien (1958). - 4. SchimasseK, H., Life Sci. 1, 629 (1962). -
5. SchinfasseK, H., Hormonwirkung an isoliert durchströmten Organen. 18. Colloquium d. Gesellschaft für physiolog. Chemie Mosbach 1967, S. 33, Springer Verlag Berlin-Göttingen-Heidelberg.

Doz. Dr. P. Glogner

355 Marburg/Lahn

Robert-Koch-Straße 7a 